Review

\title{
Is Self-Compassion Important for Health Care Practitioners?
}

Sharon Freeman Clevenger ${ }^{1,2, *}$

1. Indiana Center for Cognitive Behavior Therapy, Fort Wayne, Indiana, USA; E-Mail: sfclevenger@iccbt.org

2. Huntington University of Health Sciences, Knoxville, Tennessee, USA; E-Mail: sfclevenger@iccbt.org

* Correspondence: Sharon Freeman Clevenger; E-Mail: sfclevenger@iccbt.org

Academic Editor: Steven K. H. Aung

Special Issue: $\underline{\text { How Compassion Benefits in the Healing Process }}$

OBM Integrative and Complementary Medicine

2019, volume 4, issue 1

doi:10.21926/obm.icm.1901007
Received: November 25, 2018

Accepted: January 28, 2019

Published: January 31, 2019

\begin{abstract}
:
The cornerstone of health care practice is the provision of competent, compassionate care. Compassion is generally considered an important component in the provision of health care; however, self-compassion may be of equal importance for health care practitioners (HCP) to observe and develop. Self-compassion promotes well-being and overall mental health and may reduce fatigue and "burn-out" for HCPs [1]. Estimates of burn out in physicians and nurses range from $35-50 \%$ and is associated with emotional exhaustion, high depersonalization and cynicism, and a low sense of personal accomplishment from work [2]. In a 2014 survey of 6695 U.S. physicians in clinical practice, nearly 1 in 5 indicated an intent to reduce clinical work hours in the next year, and approximately 1 in 50 indicated that they intend to leave medicine altogether within the next 2 years to pursue a different career [3]. A troubling report found that medical students who are approaching the end of their education; which, is when the highest amount of direct patient care occurs, tend to exhibit fewer caring behaviors and less compassion and empathy in general; which are strong indicators of exhaustion and burnout [4].
\end{abstract}

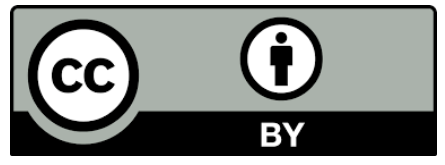

(C) 2019 by the author. This is an open access article distributed under the conditions of the Creative Commons by Attribution License, which permits unrestricted use, distribution, and reproduction in any medium or format, provided the original work is correctly cited. 
Other HCPs such as nurses, nurse practitioners and physician assistants report poor wellbeing and moderate to high levels of burnout affecting job performance and patient safety [5]. This article discusses the benefit of developing self-compassion to protect HCPs from burnout for their own well-being, and possibly for the health of their patients.

\section{Keywords}

Compassion; self-compassion; burnout; health care practitioner; clinicians

\section{Introduction}

The cornerstone of health care practice is the provision of competent, compassionate care. According to the American Medical Association Code of Ethics, the very first principle is that a "physician shall be dedicated to providing competent medical care, with compassion and respect for human dignity and rights" [6]. Compassion is one of those qualities that is generally understood, yet rarely defined. One description of compassion includes five distinct components: "recognizing suffering, understanding the universality of human suffering, feeling for the person suffering, tolerating uncomfortable feelings, and motivation to act/acting to alleviate suffering" [4]. The Merriam-Webster dictionary defines compassion as a "sympathetic consciousness of others' distress together with a desire to alleviate it", and "a feeling of wanting to help someone who is sick, hungry, in trouble, etc. [7].

"Compassion" as a human quality combines the components of "empathy" and "sympathy" with "desire to help or relieve suffering" [8]. From an evolutionary perspective, compassion was an attractive quality in a potential mate predicting increased cooperative, trustworthy behavior and mutually beneficial exchanges all qualities that are generally prized in a HCP today [8].

Compassion is generally considered an important component in the provision of health care; however, self-compassion may be of equal importance for health care practitioners (HCP) to observe and develop. Self-compassion promotes well-being and overall mental health and may reduce fatigue and "burn-out" for HCPs [1]. Estimates of burn out in physicians and nurses range from $35-50 \%$ and is associated with emotional exhaustion, high depersonalization and cynicism, and a low sense of personal accomplishment from work that may lead to leaving the profession [2]. In a 2014 survey of 6695 U.S. physicians in clinical practice, nearly 1 in 5 indicated an intent to reduce clinical work hours in the next year, and approximately 1 in 50 indicated that they intend to leave medicine altogether within the next 2 years to pursue a different career citing exhaustion and low job satisfaction as the primary reasons for leaving [3]. A troubling report found that medical students who are approaching the end of their education (when the highest amount of direct patient care occurs), tend to exhibit symptoms of exhaustion and burnout such as fewer caring behaviors and less compassion (compassion fatigue) and empathy in general [4]. Other HCPs such as nurses, nurse practitioners and physician assistants report poor wellbeing and moderate to high levels of burnout affecting job performance and patient safety [5]. Compassion fatigue (CF) often co-exists with burnout and 
includes emotional exhaustion, dysfunctional self-care and care of others hypothesized to be a consequence of prolonged exposure to suffering and stress [9]. The combination of compassion fatigue, burnout, decreased job satisfaction, interpersonal isolation and general exhaustion limits personal resources that can be used for self-compassionate actions on the part of the HCP [9].

\section{Compassion and the Autonomic Nervous}

The common denominators in compassion fatigue and burnout is stress overload that causes system exhaustion. Stress tolerance and resilience to stressors relies on a fully functioning autonomic nervous system (ANS). The ANS functions as a constantly cooperative, integrated system that enables emotion-related action tendencies. These tendencies may include fight, flight or freeze responses, or the tendency to withdraw, avoid or hide [10]. These "fight-flight" centers of the sympathetic nervous system (SNS) and the parasympathetic system (PNS) are associated with "rest and digest" or "feed and breed" functions [11]. In response to psychological stress, the ANS induces activation of sympathetic nerves along with inhibition of the PNS causing a release of adrenocortical hormones that increase heart rate, cardiac output and blood pressure [10]. Physiological processes respond to the stress response with sweating, increased breathing rate, cardiovascular and gastrointestinal arousal and musculoskeletal readiness for "fight or flight" [10]. The ability to rapidly respond to a danger or distress signal, as well as the ability to maintain high levels of readiness to act, are critically important qualities for HCPs. In healthcare workers, optimal activation and responsiveness of the stress system is essential for a sense of well-being, successful performance of tasks, and appropriate social interactions [12]. On the other hand, chronic stress with activation of the sympathetic nervous system can cause physical, behavioral and/or neuropsychiatric manifestations, such as anxiety, depression, executive and/or cognitive dysfunction, exhaustion and sleep disruption [11].

Compassion and empathy are tools used by practitioners to purposely down-regulate, or block, their pain empathy response when they need to reduce feelings of alarm or fear and increase feelings of caring and openness. This purposeful down-regulation of the stress response allows for improved capacity to be of assistance to the patient [13]. To understand the connection between stress, purposeful down-regulation of stress response and increased compassion and empathy, it may be helpful to review the psychological/physiological cascade effects of fear/stress and deactivation of fear responses. Conditioning, such as Pavlovian conditioning, shapes human reward or fear/anxiety expectations, which in turn, create physiologic responses to what we anticipate is going to happen next [11]. Soothing words, actions and behaviors of HCPs reduce negative expectations and reduce fear, anxiety and general activation or stress in patients [11]. Two studies evaluated the effect of expectation of anxiety or pain in the brains of patients given placebo based on how the placebo was introduced to them. It was discovered that both physiologic symptoms, such as pain, and activation of brain areas associated with pain or anxiety, was significantly altered based on the effect of social stimuli, such as words and rituals of therapeutic actions by clinicians $[11,14]$. In other words, the simple activities involved in words, actions and caring behavior affected the patients expectation of a negative stimuli modulating areas of the patient's brain associated with reward processing and pain 
perception [11]. It is then possibly that by employing a compassionate approach with a patient, the clinician may trigger the same pain/anxiety reduction mechanisms activated by placebos [11].

Understanding the effects of compassion toward patients on their biochemical and psychological reactions does not explain the necessity for self-compassion in the HCP. Self-compassion requires that a person treats themselves with kindness, supportive understanding, and gentleness that includes self-care and maintenance of a nonjudgmental attitude $[1,15]$. Individuals who have self-compassion also have greater social connectedness, emotional intelligence, happiness, and overall life satisfaction with less anxiety, depression, shame, and fear of failure $[1,16]$. The practice of self-compassion an ability to treat oneself kindly, with supportive understanding, and gentleness that includes self-care and maintenance of a nonjudgmental attitude toward self and others [1, 15]. Practitioners that exhibit attributes of self-compassion tend to have greater social connectedness, openness to alternative ideas, and overall life satisfaction with less anxiety, depression, shame, and fear of failure $[1,16]$. Physically, self-compassion enhances the ability to calm and self-soothe to deactivate the proinflammatory cytokine alarm pathways and neurocircuits within the ANS. Calming the ANS brain cytokine network influences every aspect of brain function relevant to the alarm/anxiety/mood/behavior interactive system that includes neurotransmitter metabolism, neuroendocrine function, synaptic plasticity, and neurocircuits that regulate mood [17]. Activation of neurocircuits in the amygdala, basal ganglia and anterior cingulate cortex due to chronic stress responses are known to trigger depression, anxiety, fatigue, psychomotor slowing, anorexia, cognitive dysfunction and sleep impairment [17]. The evidence base for the effect of calming, mindful and accepting behaviors and thoughts reduce activation of the amygdala, and the cytokine immune pathways continues to develop. Research examining brain neural circuits using functional magnetic resonance imaging ( $\mathrm{fMRI}$ ) demonstrated that compassion training increased activations in a nonoverlapping brain network spanning ventral striatum, pregenual anterior cingulate cortex and medial orbitofrontal cortex [18]. In addition, fMRI scans following compassion training evidenced by positive feelings of warmth and concern for the other correlated with increased activations in brain networks related to reward and affiliation [18]. Current knowledge regarding mindfulness, calming and accepting behaviors suggests that these components of self-compassion promote feelings of satisfaction, safety, pleasure and relaxation; which in turn, improves sleep, healing, normal appetitive drives and general well-being $[12,17,19]$. Compassion training in vulnerable populations may, therefore, represent a very potent strategy for preventing burnout.

The opposite state from stress overload is calmness. Compassion and self-compassion includes being mindfulness (calm, present and focused) of self, the situation and others [15]. Mindfulness, when combined with compassion, creates a unique neurological environment as the ANS becomes less reactive, a process that is only beginning to be understood. Some evidence of the effects of mindful compassion and meditation is emerging through neuroimaging studies that have pointed to possible involvement of the prefrontal and parietal cortices during calming exercises such as meditation [19]. Another study that evaluated mindful-meditation and neurological activity found significant changes in activity in the prefrontal cortex of the human brain [19]. Researchers used a longitudinal MRI study to investigate the relationship between changes in perceived stress in stressed but healthy adults, and changes in amygdala gray matter density following an eight-week 
mindfulness-based meditation program. At the end of the eight weeks, all participants reported reduced stress levels, and the reduced levels of perceived stress correlated positively with observed decreases in right basolateral amygdala gray matter density [19]. A second study reported observations of increased activity in the prefrontal cortex during reflective mindfulness; which in turn, correlated to decreased anxiety and improved well-being in those who practiced mindful-meditation for at least 8-weeks [20]. These studies point to possible interventions to reduce stress responses using self-calming exercises that may help to prevent negative health effects of chronic stress in HCPs.

\section{Self-Compassion and Healthcare Professionals}

Exhaustion, stress overload and burnout are emotionally negative states that threaten both compassion and self-compassion in HCPs [2]. Self-compassion in HCPs has been hypothesized to act as an emotionally positive self-attitude that should protect against the negative emotional states that includes distancing from others, reduced empathy, self-judgment, isolation, and rumination [21]. Burnout as a negative emotional state begins with a gradual loss of energy and enthusiasm leading to exhaustion, cynicism and inefficacy [2]. When a person reaches the stage of exhaustion, they unable to give anymore of oneself, become increasingly cynical and seek interpersonal distance, even avoidance, of connecting to their patients and peers $[2,21]$.

Montero-Marin and colleagues (2016) assessed self-compassion as a potential protective factor against high stress environments that lead to burnout and found that negative self-compassion dimensions play a relevant role in the development of burnout [2]. Health care practitioners are required to function effectively in, and tolerate, and even thrive in significantly stressful environments $[2,5]$. Distress tolerance as a HCP job performance skill includes the ability to tolerate difficult emotions in oneself when confronted with someone else's suffering without becoming overwhelmed by them $[4,15]$. While it is important to be empathetic and sympathetic to another's suffering, overidentification with that suffering may trigger a fight-flight response with a strong need to get away from, or in some way reduce, our awareness of their suffering [4, 15]. Decreased awareness and distancing prevent the ability to respond compassionately due to greater focus on our own discomfort; which, hinders our ability to help [4]. Psychologist Paul Gilbert stated that compassion requires the clinician to remain non-judgmental and remain accepting of, and tolerant towards, another person even when that person's condition, or response to it, gives rise to difficult feelings in oneself, such as frustration, anger, fear or disgust [15].

Montero-Marin and colleagues identified several subtypes of burnout that include 'frenetic', 'underchallenged' and 'worn-out' subtypes [2]. Each subtype correlated with negative selfcompassion dimensions explaining the burnout profiles [2]. The "frenetic" subtype correlated with negative self-judgement, "underchallenged" correlated with "isolation" and "worn-out" correlated with "over-identification" factors [2]. All three subtypes included the component of exhaustion. Exhaustion that exists with both burnout and compassion fatigue has been described as "the feeling of not being able to offer any more of oneself at work" [2]. Cynicism, added to exhaustion, accentuates interpersonal distancing attitudes towards work, patients and colleagues along with feelings of inefficacy and meaninglessness of task performance [2]. Self-care as a pre-requisite for 
self-compassion includes adequate balancing of work-life, home-life and self needs. [16]. Neff identified principle components of self-compassion to include: 1) kindness (being kind and nonjudgmental towards the self rather than self-critical), 2) mindfulness (which, like 'distress tolerance', involves holding painful feelings in mindful awareness rather than over-identifying with them), and 3) common humanity (seeing one's suffering as part of the human condition rather than as isolating) [21]. Neff indicated that self-compassion, due to its non-evaluative and interconnected nature, should counter tendencies towards narcissism, self-centeredness, and downward social comparison associated with attempts to maintain self-esteem [21]. Two very important self-care components are adequate sleep and resilience to stress [15]. In one survey of HCPs, researchers evaluated the relationship between trainable qualities (mindfulness and self-compassion), with factors conceptually related to burnout and quality of care (sleep and resilience) and found that sleep and resilience are correlated positively with both mindfulness and self-compassion [16]. Modifying negative workrelated factors, such as long duty hours, heavy work load and perceived stress (both physically and mentally), can have a significant positive effect on sleep and resilience [21]. Maintaining excessive work hours, heavy work-load and high perceived stress resulted higher rates of burnout, decreased compassion and decreased quality of care [16]. A study of compassion fatigue in oncology nurses working in community health centers found that nurses that engaged in health promotional behaviors improved the nurses well-being and counteracted the development of compassion fatigue and burnout and enhancing compassion satisfaction [22].

\section{Compassion and Self-Compassion}

Research examining the effect of health promotion behaviors as a self-compassion measure to reduce compassion fatigue and burnout links the potential positive influence that improvement of self-compassion attitudes and behaviors is likely to improve resilience to stress and increase compassion towards others [2, 21-23]. Establishing clear linkages between self-compassion in HCPs and compassionate care using comparative research studies is in the early stages of scientific discovery. In a cross-sectional study of nurses in New Zealand at risk for burnout, authors noted that greater burnout levels predicted greater barriers to compassion while self-compassion predicted fewer barriers. In addition, nurses with greater amounts of self-compassion attitudes and behaviors had lower association between burnout and burnout related barriers to compassion [24]. Two pilot studies evaluating the relationship between improved self-compassion and compassion toward others involved an eight-week mindful self-compassion training course found that participants had improved markers of self-compassion [23]. The first study examined pre- and post-test scores in selfcompassion, mindfulness, and various wellbeing outcomes among community adults, and the second was randomized controlled trial that compared a treatment group with a waitlist control group [23]. Preliminary outcomes of both studies suggested that improved self-compassion and mindfulness was significantly positively correlated with compassion towards others and improvement on scores assessing burnout factors such as life satisfaction, avoidance, depression, anxiety, and stress, with all improvements maintained at 6-month follow-up [23]. 
Negative self-expectations that increase anxiety, self-doubt and feelings of inadequacy have been hypothesized as factors that decrease self-compassion and increase stress [5]. For example, all HCPs are trained to utilize assessments and treatments that have been published in scientific journals, or in national databases, that have been validated using clinical research methodology for obvious reasons. However, for practitioners in small practices, parsing the literature can be daunting and the idea of conducting and publishing their own research is intimidating, and rarely seen as practical given competing priorities of daily life and patient demands [25]. Research is usually expected to include conventional research methodologies that rely on the gold-standard: double-blind, randomized controlled clinical trial (RCT). While this methodology may give a feeling of security and confidence regarding treatments and outcomes, the rigor under which RCT's are conducted risk loss of "face validity", external validity, and generalizability in "real world" situations. This is in large part due to overcontrolled restriction of participant variables [25]. Many people who are seen in typical "realworld" clinical practice present with numerous, complex problems, lifestyle complications and preexisting health problems [26]. Potential participants in RCT's with these common complexities present too many potentially confounding variables for a traditional RCT, and are therefore excluded from most, if not all, clinical trials [25, 26].

Exclusion of the typical complex individual from RCT's is entirely understandable; however, their exclusion from the studied population may significantly limit the utility of data obtained as far as generalizability to real world situations. Additional, over-limitation of variables as required for meaningful statistical analysis results in artificial data sets that do not reflect the people that walk through the clinic door in real practice. Practicing clinicians are left to sort through numerous studies evaluating all possible variables, usually as separate problems independently, and then try to condense the information into applicable processes for their real-world complex clients [25]. An evaluation of qualitative reports from non-scientist practitioners operating outside of academic or scientific research settings reported that they rarely research and/or publish, their own observations because they assume their data would be considered inadequate, unscientific and possibly even ridiculed [27]. Albert Ellis, the founder of Rational Emotive Therapy, encouraged HCPs to evaluate their own worth as far as their contribution to science more compassionately [28]. Ellis determined that the wealth of information held by practitioners is only accessible to others if these practitioners examine their own bias and irrational expectations regarding the value of their contributions. In other words, that practitioners exercise self-compassion (including lack of bias toward self) regarding their value to the scientific community $[28,29]$. The self-compassionate clinical practitioner understands that real world applications of health care ideally reflect a combination of the art, and science, of medicine through skillful application of scientific evidence in a creative, thoughtful manner [25].

Sometimes HCP's hold negative bias toward research outcomes and value of observations without examination of the basis for their negative expectations. For example, one area of scientific study that is emerging and controversial includes the study and practice of complementary and alternative medicine (CAM). The following example highlights the effect on practice behaviors based on probable lack of awareness of negative bias by HCPs. In the study example evaluated knowledge and attitudes of HCPs from various disciplines regarding utilization of (CAM) practices to determine if HCPs expectations affected practice attitudes and/or treatment decisions [30]. Researchers defined 
concepts and terminology for clarification at the beginning of the survey process. Complementary was defined in the study as "in addition to", while alternative was defined as or "instead of" allopathic medical care. The study included practitioners such as physicians, nurses, social workers, chiropractors, psychologists, therapists, educators and several CAM practitioners who were surveyed with "pre-" questions regarding their views of certain CAM practices and compared this to "post-" questions about actual practice behaviors. The results showed significant negative bias that contradicted actual practice behaviors likely related to negative preconceived value of CAM practices [30]. Specifically, the majority (62.5\%) of physicians reported that they did not use, prescribe or recommend CAM treatments; however, $100 \%$ of them had recommended omega-3 fatty acids along with melatonin (93.75\%), ginger or peppermint for nausea (87.50\%) [30]. Advanced practice nurses (APNs) were more comfortable with recommending CAM compared to MDs (69.89\% of APNs vs. $37 \%$ of MDs); however, many were not aware that many of the treatments they prescribed or used were actually considered CAM treatments. These results may highlight the fact that many practitioners may not identify certain treatments as CAM, be unaware of the scientific rigor for which many CAM treatments have studied, and/or believe that these CAM treatments are not within, or are beneath, their scope of practice [30]. The importance of this study regarding utilization of CAM options highlights the importance of HCPs need to evaluate their own view, and biases, of treatments in which they lack knowledge in a compassionate and self-compassionate manner. Self-compassion gives the practitioner the ability to accept limitations in knowledge in a non-judgmental manner. A self-compassionate approach is more likely to propelling them forward to seek new knowledge instead of remaining steadfast in decisions that may protect self-esteem in the short run yet limit available options. As described by Strauss, et al, decreased awareness and distancing prevent the ability to respond compassionately due to greater focus on our own discomfort; which, hinders our ability to help [4].

\section{Conclusions}

"Compassion" as a human quality combines the components of "empathy" and "sympathy" with "desire to help or relieve suffering" [8]. Self-compassion may be of equal importance to observe and develop for HCPs because it promotes well-being, overall mental and physical health, may reduce fatigue and burnout and enhance compassionate care towards others [1]. Significant risks related to a lack of self-compassion may include higher rates of compassion fatigue, burnout, decreased job satisfaction, interpersonal isolation and general exhaustion that limits personal resources that can be used for compassionate and self-compassionate actions on the part of the HCP [9]. The practice of self-compassion an ability to treat oneself kindly, with supportive understanding, and gentleness that includes self-care and maintenance of a nonjudgmental attitude toward self and others $[1,15]$. Practitioners that exhibit attributes of self-compassion tend to have greater social connectedness, openness to alternative ideas, and overall life satisfaction with less anxiety, depression, shame, and fear of failure $[1,16]$. Physically, the ability to calm and self-soothe, components of self-compassion, help to deactivate the "fight-flight" response related to chronically stressful environments which in turn reduces proinflammatory cytokine alarm pathways and neurocircuits within the ANS $[10,17]$. 
Calming, mindful and accepting behaviors and thoughts reduce activation of the cytokine immune pathways promoting feelings of satisfaction, safety, pleasure and relaxation; which in turn, promotes sleep, healing, normal appetitive drives and general well-being $[4,19]$. In contrast, lack of selfcompassion may negatively affect sleep and resilience, ultimately resulting in greater rates of burnout, decreased compassion toward others, and decreased quality of patient care [16]. Selfcompassion also gives the practitioner the ability to accept limitations or gaps in knowledge in a nonjudgmental manner propelling them forward to seek new knowledge without feeling inadequate. Increased education and training about mindfulness and self-compassion may be a viable target for future research as a way to reduce professional burnout and improve overall health in both practitioner and in turn the patients they serve [16].

\section{Acknowledgments}

The author would like to acknowledge the support of her husband, Richard for the hours of patiently waiting while various publications are created.

\section{Author Contributions}

Sharon Clevenger is sole author of this work.

\section{Competing Interests}

The authors have declared that no competing interests exist.

\section{References}

1. Eriksson T, Germundsjo L, Astrom E, Ronnlund M. Mindful self-compassion training reduces stress and burnout symptoms among practicing psychologists: A randomized controlled trial of a brief web-based intervention. Front Psychol. 2018; 9: 2340.

2. Montero-Marin J, Zubiaga F, Cereceda M, Piva Demarzo MM, Trenc P, Garcia-Campayo J. Burnout subtypes and absence of self-compassion in primary healthcare professionals: A cross-sectional study. PloS one. 2016; 11: e0157499.

3. Sinsky CA, Dyrbye LN, West CP, Satele D, Tutty M, Shanafelt TD. Professional satisfaction and the career plans of us physicians. Mayo Clin Proc. 2017; 92: 1625-1635.

4. Strauss C, Lever Taylor B, Gu J, Kuyken W, Baer R, Jones F, et al. What is compassion and how can we measure it? A review of definitions and measures. Clin Psychol Rev. 2016; 47: 15-27.

5. Hall LH, Johnson J, Watt I, Tsipa A, O'Connor DB. Healthcare staff wellbeing, burnout, and patient safety: a systematic review. PloS One. 2016; 11: e0159015.

6. AMA. AMA Principles of Medical Ethics Internet: American Medical Association; 2016. Available from: https://www.ama-assn.org/delivering-care/ama-principles-medical-ethics.

7. Merriam-Webster. Internet 2018. Compassion.

8. Goetz JL, Keltner D, Simon-Thomas E. Compassion: An evolutionary analysis and empirical review. Psychol Bull. 2010; 136: 351-374. 
9. Delaney MC. Caring for the caregivers: Evaluation of the effect of an eight-week pilot mindful self-compassion (MSC) training program on nurses' compassion fatigue and resilience. PloS One. 2018; 13: e0207261-e0207261.

10. Hasegawa M, Hayano A, Kawaguchi A, Yamanaka R. Assessment of autonomic nervous system function in nursing students using an autonomic reflex orthostatic test by heart rate spectral analysis. Biomed Rep. 2015; 3: 831-834.

11. Benedetti F, Carlino E, Pollo A. How placebos change the patient's brain. Neuropsychopharmacol. 2011; 36: 339-354.

12. Chrousos GP. Stress and disorders of the stress system. Nat Rev Endocrinol. 2009; 5: 374-381.

13. Decety J, Yang $\mathrm{CY}$, Cheng Y. Physicians down-regulate their pain empathy response: an eventrelated brain potential study. Neurolmage. 2010; 50: 1676-1682.

14. Petrovic P, Dietrich T, Fransson P, Andersson J, Carlsson K, Ingvar M. Placebo in emotional processing-induced expectations of anxiety relief activate a generalized modulatory network. Neuron. 2005; 46: 957-969.

15. Gilbert P. Mindful Compassion. United Kingdom: Robinson; 2015.

16. Kemper KJ, Mo X, Khayat R. Are mindfulness and self-compassion associated with sleep and resilience in health professionals? J Altern Complement Med. 2015; 21: 496-503.

17. Capuron L, Miller AH. Immune system to brain signaling: neuropsychopharmacological implications. Pharmacol Ther. 2011; 130: 226-238.

18. Klimecki OM, Leiberg $S$, Ricard $M$, Singer $T$. Differential pattern of functional brain plasticity after compassion and empathy training. Soc Cogn Affect Neurosci. 2014; 9: 873-879.

19. Holzel BK, Carmody J, Evans KC, Hoge EA, Dusek JA, Morgan L, et al. Stress reduction correlates with structural changes in the amygdala. Soc Cogn Affect Neurosci. 2010; 5: 11-17.

20. Barnby JM, Bailey NW, Chambers R, Fitzgerald PB. How similar are the changes in neural activity resulting from mindfulness practice in contrast to spiritual practice? Conscious Cogn. 2015; 36: 219-232.

21. Neff K. Self-Compassion: An alternative conceptualization of a healthy attitude toward oneself. Self Identity. 2003; 2: 85-101.

22. Wu S, Singh-Carlson S, Odell A, Reynolds G, Su Y. Compassion fatigue, burnout, and compassion satisfaction among oncology nurses in the united states and canada. Oncol Nurs Forum. 2016; 43: E161-169.

23. Neff KD, Germer CK. A pilot study and randomized controlled trial of the mindful self-compassion program. J Clin Psychol. 2013; 69: 28-44.

24. Dev V, Fernando AT, 3rd, Lim AG, Consedine NS. Does self-compassion mitigate the relationship between burnout and barriers to compassion? A cross-sectional quantitative study of 799 nurses. Int J Nurs Stud. 2018; 81: 81-88.

25. Clevenger SF. Conducting research as a science-based practitioner. Effect Sci Pract. 2018; 1: 7-18.

26. Jonas $W$, Lewith $G$. Toward standards of evidence for CAM research and practice. In: Walach $H$, Jones W, Lewith $G$, editors. Clinical research in complementary therapies. 2nd ed. Edinburgh: Churchill Livingstone; 2011. p. 3-42. 
27. Chambless DL. Can we talk? Fostering interchange between scientists and practitioners. Behav Ther. 2014; 45: 47-50.

28. Ellis A. Reason and emotion in psychotherapy. 2nd ed. Secaucus, NJ: Carol; 1994.

29. Corrie S, Callanan MM. Therapists' beliefs about research and the scientist-practitioner model in an evidence-based health care climate: A qualitative study. Br J Med Psychol. 2001; 74: 135-149.

30. Clevenger SF. Knowledge and attitudes towards utilizing complementary and alternative medical (CAM) treatments by mental health practitioner from various disciplines. J Tradit Complement Med. 2018. Doi: doi.org/10.1016/j.jtcme.2017.08.015

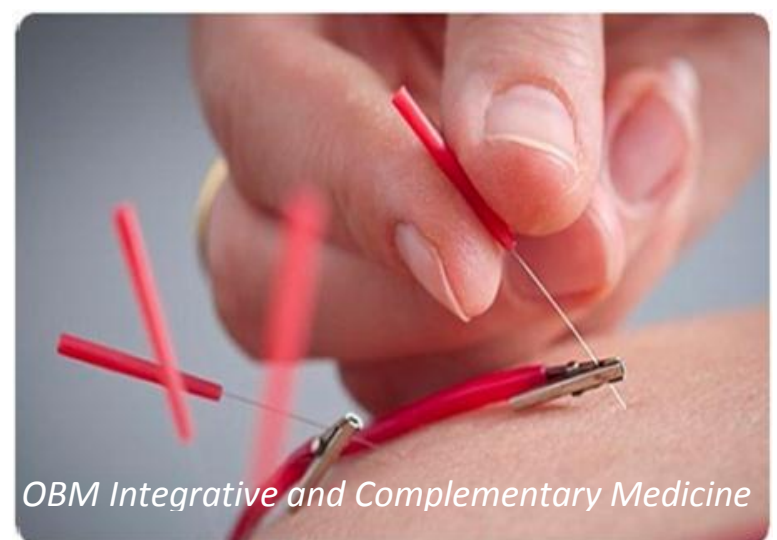

Enjoy OBM Integrative and Complementary

Medicine by:

1. Submitting a manuscript

2. Joining in volunteer reviewer bank

3. Joining Editorial Board

4. Guest editing a special issue

For more details, please visit:

http://www.lidsen.com/iournals/icm 